\title{
Presentación
}

\section{Promover la toma de decisiones en materia de salud y la dignidad inherente de los pacientes}

\section{Promoting healthcare decision making and the inherent dignity of patients}

\author{
William F. Sullivan, ${ }^{*}$ John Heng ${ }^{* *}$ \\ https://doi.org/10.36105/mye.2020v31n4.01

\section{Resumen} \\ La enseñanza moral católica afirma que es necesario el consenti- \\ miento de los pacientes para autorizar las intervenciones sanita- \\ rias que les afectan, pero no especifica las condiciones para \\ obtener dicho consentimiento o evaluar la capacidad de decisión. \\ Aquí se presentan los artículos recogidos en este número que los \\ autores han desarrollado a partir de las presentaciones que hicie- \\ ron durante un coloquio reciente de la Asociación Internacional de \\ Bioética Católica (IACB) celebrado en Quebec, Canadá. Contribuyen \\ a promover el pensamiento ético sobre la capacidad de toma de
}

\footnotetext{
* Profesor asociado del Departamento de Medicina Familiar y Comunitaria de la Universidad de Toronto; Centro Surrey Place; Equipo Académico de Salud Familiar del Hospital St. Michael, Toronto, ON, Canadá. Correo electrónico: bill.sullivan @utoronto.ca https://orcid.org/0000-0002-0520-0541

** Profesor asistente del Departamento de Filosofía y del Departamento de Estudios Interdisciplinarios, Programas de Tanatología y Estudios de la Discapacidad, King's University College de la Universidad de Western, Londres, ON, Canadá. Correo electrónico: jheng@uwo.ca https://orcid.org/0000-0002-6080-580X

Recepción: 20 de junio de 2020. Aceptación: 15 de julio de 2020.
} 
W. F. Sullivan, J. Heng

decisiones y el consentimiento. De diversas formas, piden que se respete la dignidad de todos los pacientes y que se promueva su participación tanto como sea posible en la toma de decisiones sobre su salud. Consideran que la toma de decisiones es relacional y abarca una variedad de capacidades. Examinan el papel de los miembros de la familia y de otros partidarios de la toma de decisiones en la promoción de las capacidades de los pacientes, cuya condición de salud mental o discapacidad les impide a menudo cumplir con los estándares clínicos y legales típicos para la capacidad de toma de decisiones.

Palabras clave: toma de decisiones, consentimiento, poblaciones vulnerables, toma de decisiones apoyada, autonomía relacional, participación del paciente.

Todos los artículos de este número de Medicina y Ética se refieren al respeto de la dignidad inherente de los pacientes que promueven su participación, en la medida de lo posible, en la toma de decisiones relativas a su atención médica. Estos documentos se elaboraron a partir de las ponencias presentadas en el Noveno Coloquio de la Asociación Internacional de Bioética Católica (IACB), celebrado en la ciudad de Quebec, Canadá, del 16 al 21 de junio de 2019. La Asociación Internacional de Bioética Católica (IACB) es una comunidad de especialistas en bioética y proveedores de atención de la salud y espiritualidad fundada en 2005. ${ }^{1}$ Cuarenta y ocho participantes de diferentes países tomaron parte en este coloquio, que tuvo como tema la promoción de las capacidades de las personas que necesitan apoyo para tomar decisiones en materia de atención de la salud.

Para enfocar los debates, los participantes en el coloquio consideraron cuatro grupos de pacientes: los que tienen discapacidades intelectuales y de desarrollo (por ejemplo, el síndrome de Down, el trastorno del espectro autista); las deficiencias neurocognitivas 
Promover la toma de decisiones en materia de salud y la dignidad inherente...

progresivas (por ejemplo, la enfermedad de Alzheimer, la demencia debida a la enfermedad de Parkinson); la salud mental comprometida (por ejemplo, la depresión, la esquizofrenia), y la adicción (por ejemplo, al alcohol o a otras sustancias). Esos pacientes suelen estar en la periferia de la toma de decisiones relativas a su propia atención de la salud. Los participantes también examinaron la importante función de los familiares y otros cuidadores en el apoyo a los pacientes, para que tomen decisiones relativas a la atención de su salud. Las principales conclusiones se presentan en este número, en la declaración de consenso titulada «Promover la capacidad de adoptar decisiones en materia de atención de salud». Se incluyen también algunas recomendaciones para aplicar esas conclusiones. ${ }^{2}$

Hay una larga tradición de reflexión en la bioética católica sobre el respeto al agenciamiento del paciente, la autonomía y el consentimiento. El artículo de Jos Welie ${ }^{3}$ revisa las principales ideas de esta tradición. También discute el estado moral de las directivas de cuidados anticipados, el retiro de los tratamientos de soporte vital una vez que se han iniciado, y los tratamientos involuntarios. Todos son temas sobre los que existe una variedad de puntos de vista entre los especialistas católicos en bioética. Un aspecto central de la reflexión de Jos Welie es la posición, sostenida consistentemente en la enseñanza católica sobre el cuidado de la salud, de que, sin el consentimiento, el proveedor de atención médica no está autorizado a iniciar el tratamiento. El consentimiento otorga a los proveedores de atención de la salud un derecho que no tenían antes; es decir, pasar de la benevolencia (querer el bien del paciente) a la beneficencia (hacer el bien del paciente). A partir de esto, Welie declara que involucrar activamente a los pacientes en todas las etapas de la toma de decisiones con respecto a su atención de salud es un deber ético para los proveedores de atención de la salud, basado en el respeto de la dignidad inherente de sus pacientes. Además, argumenta que es posible promover la participación del paciente dentro de un marco ético objetivo.

Medicina y Ética - Octubre-Diciembre 2020 - Vol. 31 - Núm. 4 
Este número de Medicina y Ética incluye un homenaje de Paulina Taboada al difunto cardenal Elio Sgreccia. La importante labor de Sgreccia en la promoción de la bioética personalista pone de relieve el principio ético básico de honrar la personalidad y la dignidad inherente de todos los pacientes. ${ }^{4}$ Según Sgreccia: «la participación del paciente en la gestión de su propia enfermedad y la personalización (cuando es posible) de los planes de tratamiento y de los protocolos de atención de la salud son... todos objetivos que deben perseguirse, de acuerdo con una ética que mire por la dignidad de la persona, promueva la humanización de la medicina y se esfuerce por sustituir el modelo paternalista, por el modelo de beneficencia basado en la confianza». ${ }^{5}$

¿Se aplican también estas consideraciones éticas sobre la participación de los pacientes en la adopción de decisiones relativas a su atención, a los pacientes que podrían necesitar el apoyo de otros para hacerlo? En el documento de Julian Hughes se examinan diversas cuestiones éticas que se plantean comúnmente en la atención de personas con demencia y otras deficiencias neurocognitivas progresivas. ${ }^{6}$ Él destaca tres puntos importantes que se aplican a todos esos pacientes, pero esos puntos podrían relacionarse igualmente con otros casos: 1) las cuestiones éticas en la atención de la salud entrañan la adopción de decisiones; 2) la evaluación de la capacidad de adopción de decisiones de los pacientes nunca es neutral en cuanto a los valores, y 3) el principio ético más básico en la atención de los pacientes es el de honrar su personalidad y su dignidad inherente. Al igual que Elio Sgreccia y Jos Welie, Hughes propone que el respeto de la dignidad de los pacientes implica encontrar formas de incluir e involucrar a los pacientes en la mayor medida posible en la toma de decisiones relativas a su atención. En otras palabras, este deber ético de los proveedores de atención médica se aplica a los pacientes que se consideran capaces de tomar decisiones de atención médica por sí mismos, así como a los que necesitan el apoyo de otros para hacerlo. 
Promover la toma de decisiones en materia de salud y la dignidad inherente...

El documento de Sander Welie ${ }^{7}$ describe el papel único que los defensores de los pacientes desempeñan en el sistema de salud mental holandés. En los Países Bajos, en la adopción de decisiones apoyadas en la atención de la salud mental no participan principalmente los miembros de la familia y otras personas cercanas al paciente, sino funcionarios empleados por el gobierno, a los que se denomina defensores del paciente. Esos defensores tienen el mandato de proporcionar orientación jurídica a los pacientes con respecto a sus derechos en materia de atención de la salud mental. Sander Welie señala que esos defensores no son imparciales. Al impartir asesoramiento jurídico, se espera que consideren la promoción de los mejores intereses del paciente como algo primordial. Sin embargo, esto plantea problemas éticos cuando un paciente aspira a objetivos que están conformados por un trastorno de salud mental que afecta a su pensamiento o estado de ánimo, de modo que lo que el paciente quiere no refleja la realidad de su situación o, incluso, puede ser perjudicial para él o para otros. El defensor del paciente, que a lo mejor no lo conoce muy bien, tal vez no esté en condiciones de interpretar los valores auténticos del paciente, que podrían ser diferentes de sus deseos momentáneos y transitorios. Además, el defensor del paciente está obligado a asesorarlo, dando por sentado que los deseos expresados por el paciente deben perseguirse siempre en la medida en que la ley lo permita. Sander Welie llega a la conclusión de que esta función de los defensores del paciente es éticamente problemática y angustiosa cuando, por ejemplo, el defensor se enfrenta a una petición del paciente o a una ley con la que no está de acuerdo por motivos morales, como la ley holandesa sobre la eutanasia para algunos defensores.

Christian Elia, en su documento, también habla de la eutanasia. ${ }^{8}$ Desde 2016, el suicidio asistido y la eutanasia están legalmente permitidos en Canadá. Elia acuña el término «relativismo del suicidio» para referirse a la opinión sobre el carácter moral del suicidio; es decir, el final intencional de la vida de una persona, puede variar según las razones que se utilicen para justificarlo. Los defensores 
del suicidio asistido suelen justificar esta práctica éticamente como un suicidio «racional» y lo distinguen de otros casos de suicidio, sobre los que coinciden en que la sociedad tiene el deber de prevenir. Elia sostiene que el relativismo del suicidio puede influir en la toma de decisiones de los pacientes que solicitan asistencia para poner fin a su vida. Esos pacientes también pueden buscar atención espiritual al final de la vida. Esto coloca a los proveedores de atención de la salud y a los proveedores de atención espiritual y pastoral en la posición éticamente problemática y angustiosa de tener que actuar en contra de su conciencia, si se ven obligados por la ley o guiados por directivas pastorales poco claras, a cooperar con las decisiones de esos pacientes.

En el coloquio IACB de la Junta Internacional de Fiscalización de Estupefacientes se examinó el importante papel de la familia y de otros cuidadores en la promoción de la capacidad de los pacientes para tomar decisiones. Kay Wilhelm, que tiene muchos años de experiencia clínica trabajando con pacientes con salud mental comprometida o adicciones en Australia, reflexiona sobre su experiencia con las familias que apoyan a esos pacientes en la adopción de sus decisiones en materia de atención de la salud. ${ }^{9}$ Llega a la conclusión de que, si bien algunas cuestiones para los familiares que apoyan la capacidad de sus seres queridos para tomar decisiones sobre la atención de la salud varían según el tipo de afección de que se trate, algunos principios se aplican a todos los grupos de pacientes. Por ejemplo, todos los cuidadores necesitan reconocimiento y apoyo para las funciones que desempeñan. Necesitan tener acceso a información basada en pruebas, coherentes y fidedignas, sobre las opciones disponibles, la mejor manera de cumplir sus funciones de apoyo a la adopción de decisiones y sobre la forma de cuidar de su propia salud y bienestar, a fin de atender a la persona a la que están cuidando.

El artículo de Marie-Jo Thiel presenta una importante perspectiva ética sobre la toma de decisiones en materia de salud desde 
Promover la toma de decisiones en materia de salud y la dignidad inherente...

un contexto no angloamericano. ${ }^{10}$ Elaborando las ideas de Paul Ricoeur, Thiel, al igual que Sullivan, Heng y Bach, entiende el agenciamiento y la autonomía del paciente como algo relacional, pero por una razón diferente y complementaria. Escribe: «Consentir es 'captar intuitivamente, de manera sensible', no sólo lo que está en juego en una situación clínica conmigo mismo (es decir, mis opiniones y mis creencias), sino también con los demás, para aceptar una propuesta de tratamiento médico que no perturbe mi existencia, con el médico que informa, los miembros de la familia y otros cuidadores, porque ellos también forman parte de mi existencia». Esta idea subyace en su análisis de las difíciles decisiones éticas relativas a la retirada de los tratamientos de mantenimiento de la vida en los pacientes que están inconscientes, pero que no tienen instrucciones anticipadas o cuyas instrucciones no están claras. Aunque la ley francesa, a diferencia de las leyes de los países angloamericanos, designa a los proveedores de atención médica, y no a los miembros de la familia u otros suplentes que toman decisiones por el paciente, como los encargados de tomar las decisiones finales en tales situaciones, Thiel sostiene que la atención médica al final de la vida debe estar centrada en la familia. Los familiares de los pacientes se verán inevitablemente afectados por las decisiones de retirar los tratamientos de mantenimiento de la vida. Insta a que se optimice la comunicación entre los proveedores de atención de la salud y las familias de los pacientes, respondiendo a sus preocupaciones con respecto al cuidado de su ser querido y atendiendo a sus necesidades derivadas de la perspectiva de perder a un ser querido. Thiel propone que la muerte es un momento único y definitivo, y a menudo la experiencia de los familiares con el paciente condiciona tanto el proceso de toma de decisiones en relación con el cuidado de su ser querido al final de la vida, como el alcance del trauma relacionado con su propio proceso de duelo.

Los artículos de este número de Medicina y Ética presentan contribuciones prácticas e invitan a la reflexión sobre el pensamiento 
bioético en lo que respecta a la toma de decisiones en materia de salud. Son sustanciales tanto en la amplitud como en la profundidad de la reflexión. Destacan la importancia no sólo de implicar a los pacientes en la medida de lo posible en la toma de decisiones relativas a su atención, sino también de ofrecer alojamiento y ayuda a los responsables de la toma de decisiones que puedan necesitar para cualquier aspecto del proceso de toma de decisiones. En los artículos se destaca también el papel fundamental de los familiares que prestan atención a los pacientes y de otras personas que apoyan la adopción de decisiones que, a su vez, necesitarán una buena comunicación, atención y apoyo de los proveedores de atención de la salud para desempeñar ese papel. La asociación entre los pacientes, sus familiares, otros apoyos y los proveedores de atención médica es lo que mejor fomenta la capacidad de decisión de los pacientes y su agenciamiento y autonomía. Éstos son inherentemente relacionales.

Estos documentos nos dejan con muchas preguntas bioéticas para explorar. También plantean un desafío práctico para todos: hay que fortalecer las asociaciones de atención de salud como se describen anteriormente, y encontrar formas de fomentar las amistades y otras relaciones estrechas con quienes están socialmente aislados, y carecen de personas de confianza y cercanas en su vida que puedan apoyarlos en la toma de decisiones de atención de salud cuando necesitan ese apoyo.

\section{Notas bibliográficas}

${ }^{1}$ Para información sobre la IACB, véase: http://iacb.ca.

${ }^{2}$ Sullivan WF, Heng J, DeBono C, et al. Promoviendo la capacidad de tomar decisiones sobre el cuidado de la salud. Declaración de consenso del $9^{\circ}$ Coloquio Internacional de la IACB. National Catholic Bioethics Quarterly. Verano del 2020; 20(2): 617-633. https://doi.org/10.16925/greylit.1514

${ }^{3}$ Welie JVM. Agenciamiento del paciente, autonomía y consentimiento. Medicina y Ética. Octubre de 2020; 31(4). 
Promover la toma de decisiones en materia de salud y la dignidad inherente...

4 Taboada P. Una vida para la Bioética. Homenaje al cardenal Elio Sgreccia (1928-2019) tras su muerte. Medicina y Ética. Octubre de 2020; 31(4). https:// doi.org/10.4081/mem.2016.479

${ }^{5}$ Sgreccia E. DiCamillio JA, Miller MJ, traductores. Bioética personalista. Fundamentos y aplicaciones. Filadelfia, EE.UU. The National Catholic Bioethics Center; 2012. Citado en Welie JVM. Agencia del paciente, autonomía y consentimiento. Medicina y Ética. Octubre de 2020; 31(4).

${ }^{6}$ Hughes JC. Cuestiones éticas que se plantean en la toma de decisiones en materia de atención de la salud de las personas que viven con demencia y otros trastornos cognitivos progresivos. Medicina y Ética. Octubre de 2020;31(4). https://doi.org/10.35537/10915/5429

${ }^{7}$ Welie SPK. Defensa de los pacientes psiquiátricos en los Países Bajos: El modelo de la Fundación Nacional Holandesa de Defensores del Paciente en el Cuidado de la Salud Mental. Medicina y Ética. Octubre de 2020; 31(4). https://doi.org/ $10.4067 / \mathrm{s} 1726-569 \times 2012000200006$

${ }^{8}$ Elia CD. Cuestiones morales subyacentes a la orientación pastoral y la práctica sacramental para pacientes que eligen el suicidio o la eutanasia asistida: el dilema canadiense. Medicina y Ética. Octubre de 2020; 31(4).

${ }^{9}$ Wilhelm K. Decision making in mental health care: the caregiver's role. Medicina y Ética. Octubre de 2020; 31(4).

${ }_{10}$ Thiel M-J. Rechazar y retirar el tratamiento al final de la vida: Complejidades éticas que implican a los pacientes que carecen de capacidad de decisión. Medicina y Ética. Octubre del 2020; 31(4). 
